\title{
Atomically Layered and Ordered Rare-Earth i- MAX Phases: A New Class of Magnetic Quaternary Compounds
}

Quanzheng Tao, J un Lu, Martin Dahlqvist, Aurelija Mockuté, Stuart Calder, Andrejs Petruhins, Rahele Meshkian, Oleg Rivin, Daniel Potashnikov, Elad N. Caspi, Hagai Shaked, Andreas Hoser, Christine Opagiste, Rose-Marie Galera, Ruslan Salikhov, Ulf Wiedwald, Clemens Ritter, Andrew R. Wildes, Boerje J ohansson, Lars Hultman, Michael Fade, Michel Barsoum and J ohanna Rosén

The self-archived postprint version of this journal article is available at Linköping University Institutional Repository (DiVA):

http:/ / urn.kb.se/ resolve?urn=urn:nbn:se:liu:diva-157225

N.B.: When citing this work, cite the original publication.

Tao, Q., Lu, J ., Dahlqvist, M., Mockuté, A., Calder, S., Petruhins, A., Meshkian, R., Rivin, O., Potashnikov, D., Caspi, E. N., Shaked, H., Hoser, A., Opagiste, C., Galera, R., Salikhov, R., Wiedwald, U., Ritter, C., Wildes, A. R., J ohansson, B., Hultman, L., Fade, M., Barsoum, M., Rosén, J ., (2019), Atomically Layered and Ordered Rare-Earth i-MAX Phases: A New Class of Magnetic Quaternary Compounds, Chemistry of Materials, 31(7), 2476-2485.

https:// doi.org/ 10.1021/ acs.chemmater.8b05298

Original publication available at:

https:/ / doi.org/ 10.1021/acs.chemmater.8b05298

Copyright: American Chemical Society

http:// pubs.acs.org/ 


\title{
Atomically Layered and Ordered Rare-Earth i-MAX Phases: A New Class of Magnetic Quaternary Compounds
}

\author{
Quanzheng Tao ${ }^{1}$, Jun Lu1 ${ }^{1}$, Martin Dahlqvist ${ }^{1}$, Aurelija Mockute ${ }^{1,2}$, Stuart Calder ${ }^{2}$, Andrejs \\ Petruhins ${ }^{1}$, Rahele Meshkian¹, Oleg Rivin ${ }^{3,4}$, Daniel Potashnikov,6, El'ad Caspi ${ }^{3}$, Hagai Shaked7, An- \\ dreas Hoser ${ }^{4}$, Christine Opagiste ${ }^{8}$, Rose-Marie Galera ${ }^{8}$, Ruslan Salikhov ${ }^{9}$, Ulf Wiedwald ${ }^{9}$, Clemens \\ Ritter $^{10}$, Andrew R. Wildes ${ }^{10}$, Börje Johansson ${ }^{11,12}$, Lars Hultman ${ }^{1}$, Michael Farle ${ }^{9}$, Michel W. Bar- \\ soum $^{1,13}$, Johanna Rosen ${ }^{1 *}$
}

1Thin Film Physics Division, Department of Physics, Chemistry, and Biology (IFM), Linköping University, SE-581 83 Linköping, Sweden

${ }^{2}$ Neutron Scattering Division, Oak Ridge National Laboratory, Oak Ridge, Tennessee 37831, USA

${ }^{3}$ Physics Department, Nuclear Research Centre - Negev, P. O. Box 9001 Beer-Sheva 84190, Israel

${ }^{4}$ Helmholtz-Zentrum Berlin fur Materialen und Energie, Berlin 14109, Germany

${ }^{5}$ Faculty of Physics, Technion-Israeli Institute of Technology, Haifa 32000, Israel

${ }^{6}$ Israel Atomic Energy Commission, P. O. Box 7061, Tel-Aviv 61070, Israel

${ }^{7}$ Physics Department, Ben-Gurion University of the Negev, P. 0. Box 653 Beer-Sheva 84105, Israel

${ }^{8}$ Institut Neel, CNRS, Univ. Grenoble Alpes, Grenoble INP, FR-38000 Grenoble, France

${ }^{9}$ Faculty of Physics and Center for Nanointegration (CENIDE), University of Duisburg-Essen, 47057 Duisburg, Germany

${ }^{10}$ Institut Laue-Langevin, B P 156, 38042 Grenoble Cedex 9, France

${ }^{11}$ Department of Physics and Astronomy, Uppsala University, Box 516, SE-751 20 Uppsala, Sweden

${ }^{12}$ Humboldt University, Physics Department, Zum Grossen Windkanal 6, D-12489 Berlin, Germany

${ }^{13}$ Department of Materials Science and Engineering, Drexel University, Philadelphia, Pennsylvania 19104, USA.

ABSTRACT: In 2017, we discovered quaternary $i$-MAX phases - atomically layered solids, where M is an early transition metal, $A$ is an A group element, and $\mathrm{X}$ is $\mathrm{C}$ - with a $\left(\mathrm{M}^{1}{ }_{2 / 3} \mathrm{M}^{2}{ }_{1 / 3}\right){ }_{2} \mathrm{AC}$ chemistry, where the $\mathrm{M}^{1}$ and $\mathrm{M}^{2}$ atoms are in-plane ordered. Herein we report on the discovery of a class of magnetic $i$-MAX phases in which bi-layers of a quasi-2D magnetic frustrated triangular lattice overlays a Mo honeycomb arrangement and an Al Kagomé lattice. The chemistry of this family is $\left(\mathrm{Mo}_{2 / 3} \mathrm{RE}_{1 / 3}\right)_{2} \mathrm{AlC}$, and the rare earth elements, $\mathrm{RE}$, are Ce, Pr, Nd, Sm, Gd, Tb, Dy, Ho, Er, Tm, and Lu. The magnetic properties were characterized and found to display a plethora of ground states, resulting from an interplay of competing magnetic interactions in the presence of magnetocrystalline anisotropy.

Rare earth, RE, containing solids exhibit a variety of magnetic and electronic ground states through hybridization between their $4 f$ and conduction electrons ${ }^{1-3}$. Heavy fermion compounds, mostly based on $\mathrm{Ce}$ and $\mathrm{Yb}$, are prototype systems for the study of quantum critical ${ }^{4}$ and collective quantum states ${ }^{5}$. Other degrees of freedom, like orbital and valence, yield a variety of states of matter ${ }^{6,7}$. For example, Sm exhibits valence instabilities that play a significant role in the exotic, strongly correlated, behavior in Sm compounds such as $\mathrm{SmOs}_{4} \mathrm{Sb}_{12}{ }^{8}$ and $\mathrm{SmB}_{6}{ }^{9}$.

While the collective magnetic response of RE ions is also influenced by crystal structure and chemical environment, we propose to probe for such effects in the $M_{n+1} A X_{n}$ phases, where $\mathrm{M}$ is an early transition metal (that as shown here can be partially substituted with a RE), A is an A-group element (mostly 13 and 14) and X is C and/or N. These so called MAX phases are a class of atomically laminar materials. Most common examples are from carbides and nitrides ${ }^{10}$, combining the characteristics of metals and ceramics ${ }^{11}$. The rich chemistry of these materials renders them promising for applications ranging from structural materials at extreme conditions $^{12}$, Ohmic contact materials for semiconductors ${ }^{13}$, and as precursors for their 2D counterparts, i.e. MXenes ${ }^{14,}$ 15 .

Recently, we discovered quaternary $\left(\mathrm{M}^{1}{ }_{2 / 3}, \mathrm{M}^{2}{ }_{1 / 3}\right)_{2} \mathrm{AC}$ phases, that we coined $i$-MAX because the M elements are in-plane ordered. The $\mathrm{M}^{1}$ atoms sit on a honeycomb lattice and the $\mathrm{M}^{2}$ on a triangular lattice. The A layers form Kagomé-like patterns. This discovery has greatly expanded the number of $\mathrm{M}$ elements and their combinations that can be incorporated in the MAX phases. Examples include $\left(\mathrm{Mo}_{2 / 3} \mathrm{Sc}_{1 / 3}\right)_{2} \mathrm{AlC},\left(\mathrm{Mo}_{2 / 3} \mathrm{Y}_{1 / 3}\right)_{2} \mathrm{AlC},\left(\mathrm{V}_{2 / 3} \mathrm{Zr}_{1 / 3}\right)_{2} \mathrm{AlC}^{16,17}$ and $\left(\mathrm{W}_{2 / 3} \mathrm{Sc}_{1 / 3}\right)_{2} \mathrm{AlC}^{18}$. Furthermore, selective etching of these 
phases allows for atomic scale engineering of ordered vacancies in the 2D nanosheet ${ }^{16,18 .}$

Herein, we report on the synthesis of 11 new $i$-MAX phases with the general formula $\left(\mathrm{M}^{1}{ }_{2 / 3}, \mathrm{M}^{2}{ }_{1 / 3}\right)_{2} \mathrm{AlC}$, where $\mathrm{M}^{1}$ is $\mathrm{Mo}$ and $\mathrm{M}^{2}$ is a RE element, viz. Ce, $\mathrm{Pr}, \mathrm{Nd}, \mathrm{Sm}, \mathrm{Gd}, \mathrm{Tb}$, Dy, Ho, Er, Tm, and Lu. In all cases, the resulting structure is layered, with chain-like arrangement of the RE element (Fig. 1a). When the metal layers are imaged from the top, it is clear that the RE atoms (depicted in blue in Fig. 1b) form a 2D triangular lattice. The here demonstrated frustrated quasi-2D sheets of RE elements are expected to lead to complex magnetic properties via intra- and interplane exchanges caused by the well-known Ruderman-KittelKasuya-Yosida (RKKY) indirect coupling mechanism of localized inner $4 f$-shell electron spins through the conduction electrons. As shown here, the interplay of competing magnetic interactions in the presence of magnetocrystalline anisotropy gives rise to different magnetic properties. The purpose of this work is to describe the synthesis, structural and magnetic characteristics of these new $i$-MAX phases.

\section{Materials and Methods}

Material synthesis

Polycrystalline $\left(\mathrm{Mo}_{2 / 3} \mathrm{RE}_{1 / 3}\right)_{2} \mathrm{AlC}$ samples were synthesized by pressureless sintering the following elemental powders: graphite (99.999\%), Mo (99.99\%) from Sigma-Aldrich, and Al (99.8\%) from Alfa Aesar, and RE (99.9\%) from Stanford Advanced Materials. Stoichiometric amounts of the elemental powders were manually mixed in an agate mortar, placed in an alumina crucible that was in turn inserted in an alumina tube furnace through which $5 \mathrm{sccm}$ of Ar was flowing. The furnace was then heated, at $5^{\circ} \mathrm{C}$ per minute, up to $1500{ }^{\circ} \mathrm{C}$ and held at $1500{ }^{\circ} \mathrm{C}$ for $10 \mathrm{~h}$, before furnace cooling to room temperature (RT). The loosely sintered powders were crushed into a fine powder that was directly used for further analysis.

\section{Structural characterization}

X-ray diffraction (XRD) was carried out on a PANalytical $X^{\prime}$ Pert powder diffractometer, with a $\mathrm{Cu}$ source $\left(\lambda_{\mathrm{K}_{\alpha}} \approx 1.54 \AA\right)$. Scanning transmission electron microscopy (STEM) combined with high angle annular dark field imaging (STEM-HAADF) and energy-dispersive X-ray spectroscopy (EDX) analysis with a Super-X EDX detector was performed in the double-corrected Linköping FEI Titan ${ }^{3}$, operated at $300 \mathrm{kV}$. Selected area electron diffraction (SAED) was performed on a FEI Tecnai T20 TEM operating at $200 \mathrm{kV}$.

\section{Magnetization measurements}

The powders' magnetic properties were examined in magnetic fields up to $\pm 9 \mathrm{~T}$ at variable temperatures using a vibrating sample magnetometer (VSM) in a Quantum Design DynaCool Physical Property Measurement System (PPMS).

\section{Heat capacity measurements}

In the 2-100 K temperature range, the specific heat $\left(c_{p}\right)$ was measured using the relaxation method with a Quantum Design PPMS. The transition temperatures were deduced from the inflexion points of the anomalies. A tentative determination of the magnetic entropy $\left(\mathrm{S}_{\mathrm{M}}\right)$ from the experimental $c_{p}$ was performed for $\mathrm{RE}=\mathrm{Tb}$. This was done to determine the degeneracy of the magnetic ground state. It is expected that the crystal electric field, CEF, interactions partly lift the $2 \mathrm{~J}+1$ degeneracy of the fundamental multiplet. Thus, it is necessary to properly subtract the phonon contribution from the total $c_{p}$ in order to obtain the magnetic contribution, $\mathrm{c}_{\mathrm{mag}}$. For these compounds the phonon contribution has been determined from $c_{p}$ curves measured for the non-magnetic $\left(\mathrm{Mo}_{2 / 3} \mathrm{Lu}_{1 / 3}\right)_{2} \mathrm{AlC}$. The $\mathrm{S}_{\mathrm{M}}$ was obtained by integrating $\mathrm{c}_{\mathrm{mag}} / \mathrm{T}$ from $0 \mathrm{~K}$ to $\mathrm{T}$. Since the lowest temperature reached in the present measurements was $2 \mathrm{~K}$, it is likely that $S_{M}$ is underestimated here.

Neutron powder diffraction

Neutron powder diffraction (NPD) measurement of $\left(\mathrm{Mo}_{2 / 3} \mathrm{~Tb}_{1 / 3}\right)_{2} \mathrm{AlC}$ were carried out using the HB-2A highflux powder diffractometer at the High Flux Isotope Reactor at Oak Ridge National Laboratory (ORNL, USA). A 5 g powder sample was loaded in an Al cylindrical sample holder. Measurements were performed with a $\lambda=2.41 \AA$ neutron wavelength produced by (113) reflections from a vertically focusing $\mathrm{Ge}$ monochromator. $\left(\mathrm{Mo}_{2 / 3} \mathrm{~Tb}_{1 / 3}\right)_{2} \mathrm{AlC}$ was measured at $1.5 \mathrm{~K}, 8 \mathrm{~K}, 15 \mathrm{~K}, 22 \mathrm{~K}$, and $100 \mathrm{~K}$. The data were collected using a ${ }^{3} \mathrm{He}$ detector bank covering a $2 \theta$ range of $7^{\circ}-$ $133^{\circ}$ in steps of $0.05^{\circ}$. The magnetic structures were determined using the following procedure: The additional peaks below the ordering temperature were fitted by a gaussian function to find the peak position, and corrected for zerooffset. The propagation vectors were searched for using Fullprof "k-search". The symmetry analysis was performed by the Fullprof "BasIreps". Finally, the spin configuration was determined by considering all possible magnetic models.

The high flux E6 neutron diffractometer, located at the Helmholtz Zentrum Berlin (HZB, Germany), was used to obtain NPD profiles of ( $\left.\mathrm{Mo}_{2 / 3} \mathrm{Er}_{1 / 3}\right)_{2} \mathrm{AlC}$ at 298(2), 22(1) and $1.5(3) \mathrm{K}$. An incident beam $(\lambda \sim 2.4 \AA)$ was obtained using a focusing pyrolytic graphite monochromator. A $4 \mathrm{~g}$ powder sample was loaded into a $6 \mathrm{~mm}$ diameter vanadium, $\mathrm{V}$, cylindrical sample holder. The determination of the magnetic structure was performed using the SARAh package followed by a symmetry analysis with the BasIreps code. A systematic scan of the special propagation vectors in the Brillouin zone, followed by a search along symmetry lines and planes combined with a Monte Carlo refinement of the magnetic structure was performed until a suitable propagation vector was found. Finally, the refinement was performed on the 1.5 $\mathrm{K}$ data by considering all possible magnetic models.

\section{Theoretical calculations}

All calculations are performed within the framework of density functional theory (DFT) as implemented in the Vienna $a b$-initio simulation package (VASP) ${ }^{23-25}$ using the projector augmented wave method ${ }^{26}, 27$ with spin-polarized generalized gradient approximation (GGA) as parameterized by Perdew-Burke-Ernzerhof (PBE) ${ }^{28}$ for treating electron exchange and correlation effects. Wave functions were expanded in plane waves up to an energy cut-off of $400 \mathrm{eV}$, and the sampling of the Brillouin zones was carried out using the Monkhorst-Pack scheme ${ }^{29}$. We used two different treatment of the $4 f$ electrons; (i) in the core, denoted $4 f$-core throughout this paper, or (ii) as part of the valence band, denoted $4 f$-band throughout this paper. For the latter case, the degree of localization of the $4 f$ electrons is important and needs to be considered. As a first approximation we therefore used the rotationally invariant approach as proposed by Dudarev et al. ${ }^{30}$ Note that within this formalism the onsite Coulomb parameter, $U$, and the exchange 
parameter, $J$, are spherically averaged into a single effective interaction parameter $U_{\text {eff }}=U-J$, which does not depend on their individual values. Since the degree of localization for the $4 f$ electrons is unknown we chose to use different values of $U_{\text {eff }}$ over the lanthanide series with a focus on $U_{\text {eff }}=3$ or $7 \mathrm{eV}$. As a first approximation, we modeled the magnetism in $\left(\mathrm{Mo}_{2 / 3} \mathrm{RE}_{1 / 3}\right)_{2} \mathrm{AlC}$ within a collinear framework. The collinear magnetic spin configurations considered are visualized in Supplementary Fig. S4. The equilibrium structures are obtained by minimization of the total energy with respect to volume and with full relaxation of atomic positions and unit cell parameters until forces are converged below $10^{-3} \mathrm{eV} \AA^{-1}$.

\section{Results}

Crystal structure and in-plane chemical order

The synthesis of these phases is summarized in the Methods Section. STEM and SAED were used for structural characterization and space group identification. XRD Rietveld refinements were used to determine the crystal structures and sample purity. Neutron powder diffraction (NPD) was used to correlate crystallographic and magnetic structures in selected compounds. Three polymorphs were identified with symmetries $C 2 / c, C 2 / m$, and $C m c m$, represented by the $\mathrm{Tb}, \mathrm{Ce}$, and Gd, $i$-MAX phases, respectively. Henceforth, for the sake of brevity, the various phases will simply be referred to by their RE element.

The Tb phase was the one with highest sample purity and was chosen to depict the $C 2 / c$ structure. Figure 1 c-f show STEM images of Tb along [100], [010], [110], and [001] zone axes, respectively. The $\mathrm{Tb}$ atoms appear brightest due to their large atomic number ( $\mathrm{Z}$ contrast); $\mathrm{Mo}$ and $\mathrm{Al}$ are less bright; the lightest element, $\mathrm{C}$, is not visible. The structure viewed along [100] look identical to a traditional MAX phase viewed along [11-20]. In-plane Mo and Tb chemical ordering is evidenced by the [010] and [110] zone axes, with the $\mathrm{Tb}$ atoms extending from the M-layers towards the Al-layers; a general feature of most $i$-MAX phases ${ }^{16}$. Furthermore, the non-uniform $\mathrm{Al}$ atomic distribution, (Fig. 1g) originates from a Kagomé-like ordering in the Al layer. The SAED patterns (insets in Fig. 1c-f) are consistent with monoclinic symmetry. The atomic arrangements, characteristic of monoclinic symmetry, determined by DFT, are shown as insets on the right of Fig. 1c-f. The agreement between the STEM images and those predicted by DFT are excellent, confirming the chosen structure. The atomic arrangement of the $\mathrm{Mo}, \mathrm{Tb}$, and $\mathrm{Al}$ atoms is revealed in real space in the atomic resolved EDX mapping of these elements, shown on right of Fig. 1g-h. In the [110] map (Fig. 1g), the green dots represent individual Tb columns; in the [001] map (Fig. 1f), they represent four Tb columns.

Rietveld refinement of the XRD pattern of the Tb phase (Fig. 1i) confirmed the $C 2 / c$ structure, see Table S1. NPD pattern of the same phase at $100 \mathrm{~K}$, (Fig. 1j) is also fully consistent with the XRD and STEM analyses and reveal full occupancy of the Mo and Tb sites with no intermixing. We thus conclude that the $\mathrm{Tb}$ atoms form a close to perfect triangular lattice.

The STEM images of the Ce phase (Fig. 2a-c) are consistent with a $C 2 / m$ symmetry. Similar to $\mathrm{Tb}$, the Ce atoms appear brightest, with Ce extending from the M-plane towards Al. Lastly, the STEM images of the Gd phase, (Fig. 2de) are consistent with a $\mathrm{Cmcm}$ symmetry, and display the same characteristic features as its $\mathrm{Tb}$ and Ce counterparts.
STEM images of the Pr, Nd, Sm, Gd, Dy, Ho, Er, Tm, and Lucontaining $i$-MAX phases are shown in Fig. S1. The XRD results are shown in Fig. $\mathbf{S} 2$ and the sample/structural information from refinement is listed in Tables S1, S2 in Section S1, respectively.

Figure 3 summarizes our results: The Ce and Pr-containing $i$-MAX phases crystalize in the $C 2 / m$ structure; the $\mathrm{Tb}$, $\mathrm{Nd}, \mathrm{Gd}, \mathrm{Dy}, \mathrm{Ho}, \mathrm{Er}$, and Tm phases are $C 2 / c$. The Gd phase crystallizes in both $\mathrm{Cmcm}$ and $C 2 / \mathrm{c}$. For the Sm phase $C 2 / \mathrm{m}$ and $C 2 / c$ structures co-exist. Note that the $C 2 / m$ structure is preferred for the lighter elements (to the left in Fig. 3), while $C 2 / c$ is preferred by the heavier ones (right).

Figures 4a-d compare the experimental (solid circles) and DFT predicted (crosses and triangles) lattice parameters, LPs, $a, b$, and $c$ and the monoclinic angle $\beta$, for the nine phases with space group $C 2 / c$, showing that the LPs decrease with increasing $4 f$ electrons. This behavior is in line with the lanthanide contraction ${ }^{31,32}$. The monoclinic angle $\beta$ is rather constant at $\sim 103.5^{\circ}$.

As noted above, the DFT calculations were carried out with one of two assumptions. The first treats the $4 f$ electrons as part of the core (red crosses in Fig. 4a-d). The second treats the $4 f$ electrons as a band (inverted blue triangles in Fig. 4a-d). In both cases, the overall agreement between theory and experiment is good for the $a$ and $b$ LPs. Theory, however, overestimates the $c$-LPs and angle, $\beta$ (Fig. 4c and d). The absence of data for $\mathrm{Eu}, \mathrm{Yb}$ and Pm reflects the fact that they were not realized experimentally.

The $4 f$-band model reproduces the LP trend well, with the largest relative difference for $c(-0.1$ to $+0.9 \%)$ compared to the experimental values. As a first approximation, collinear spin configurations are assessed, and for a majority of the RE elements (Ce, Pr, Nd, Sm, Gd, Dy, Ho, Er, Tm) different spin-states are accessible. For each spin-state, the initially assigned spin configurations, shown in Fig. S4, are degenerate in energies within $2 \mathrm{meV} /$ atom. Calculated structural parameters are therefore presented in Fig. 4a-d as average, maximum, and minimum values for the degenerate spin configurations corresponding to the spin-state of lowest energy. The maxima and minima are represented by a blue band in Fig. 4a-d. A closer examination of the calculated local magnetic moments of the RE elements, shown in Fig. S5 for attained spin-states from the $4 f$-band model, show the largest moments for $\operatorname{Gd}\left(7 \mu_{\mathrm{B}}\right)$.

\section{Magnetic properties}

The temperature $(\mathrm{T})$ and magnetic field $(\mathrm{H})$ dependencies of the magnetization (M) of the Ce, Pr, Nd, Sm, Gd, Tb, Dy, Ho, Er, and Tm phases were measured. Except for Sm and $\mathrm{Tm}$, all compounds show long range magnetic order at temperatures ranging from $3.6 \mathrm{~K}$ for Er, to $28 \mathrm{~K}$ for $\mathrm{Tb}$ (see Tab. 1). Fig. $\mathbf{S 6}$ plots the inverse susceptibility $1 / \chi$ (deduced from Arrott plots ${ }^{33}$ ) or $\mathrm{H} / \mathrm{M}$ vs. $\mathrm{T}$ for the 9 phases. In all cases but $\mathrm{Sm}$, the transition to a magnetically ordered states follow a Curie-Weiss (C-W) law of the form

$$
\chi=\mathrm{C} /(\mathrm{T}-\theta)
$$

where $\mathrm{C}$ is the Curie constant, and $\theta$ is the $\mathrm{C}-\mathrm{W}$ temperature. From the slopes, the effective moment $\mu_{\text {eff }}$ per $R E$ atom is estimated (see Section S4). The parameters deduced from magnetometry are summarized in Tab. 1. The C-W temperatures vary, with the highest absolute values of about $20 \mathrm{~K}$ for $\mathrm{Gd}$. The effective magnetic moments $\mu_{\text {eff }}$ per $\mathrm{RE}$ atom rise from $3.6 \mu_{\mathrm{B}}$ for Pr to $10.5 \mu_{\mathrm{B}}$ for Dy and then 
decreases to $\mu_{\text {eff }}=7.32 \mu_{\mathrm{B}}$ for Tm. For most phases, the Weiss temperatures are negative, reflecting antiferromagnetic interaction in these phases. Magnetization at 9T shows similar trends with the effective moments. Generally, $\mathrm{M}$ is much reduced compared to theoretical values. For some materials, like $\mathrm{Tb}$ and $\mathrm{Er}$, the reduction is attributed to a $\mathrm{CEF}$ effect. For Tb, Dy, and Gd, the Ms do not reach saturation at 9T. The M of Sm is extremely weak. The magnetic behavior of possible impurities are summarized in Section S1, to exclude their potential contribution to the measured data.

To exemplify the diversity and richness of the magnetic responses, four compounds, $\mathrm{Nd}, \mathrm{Gd}, \mathrm{Tb}$, and Er were selected for a more detailed magnetic analysis.

Each is presented separately in what follows.

$\left(\mathbf{M o}_{2 / 3} \mathbf{N d}_{1 / 3}\right)_{2}$ AlC: In Fig. 5a, the M/H vs. T curve displays a distinct peak labelled $\mathrm{T}_{\mathrm{N}}$ at $7.6 \mathrm{~K}$, indicating a phase transition. Consistent with the magnetization data, a pronounced lambda anomaly in the heat capacity, $\mathrm{c}_{\mathrm{p}}$, is observed at $\sim 8 \mathrm{~K}$ (right y-axis in Fig. 5a). Plots of $\mathrm{M}(\mathrm{H})$ are shown in inset of Fig. 5a. While at higher temperature, M shows Langevin-type paramagnetic behavior, there is a field induced transition below $\mathrm{T}_{\mathrm{N}}$ at around $1 \mathrm{~T}$. The magnetization at $3 \mathrm{~K}$ reaches $\sim 1.6 \mu_{\mathrm{B}} / \mathrm{Nd}$ at $9 \mathrm{~T}$, which is about half of the theoretical saturated moment $\left(3.27 \mu_{\mathrm{B}}\right)$. The reduced moment is likely attributed to the CEF effect ${ }^{34}$. From the results shown in Fig. S6b, the effective moment in the paramagnetic state is estimated to be $\mu_{\text {eff }}=3.73(3) \mu_{\mathrm{B}}$. This is close to the free ion value of $\mathrm{Nd}^{3+}\left(3.62 \mu_{\mathrm{B}}\right)$. The extrapolated negative temperature $\theta=-9.3 \mathrm{~K}$ indicates AFM correlation between the $\mathrm{Nd}$ atoms.

(Mo M/3 $\left._{\mathbf{3}} \mathbf{G d}_{1 / 3}\right)_{2}$ AlC: The H/M curve measured under $0.1 \mathrm{~T}$ together with the inverse susceptibility $(1 / \chi)$ deduced from Arrott plots are shown in Fig. S6d. Both curves are consistent with a C-W law above $50 \mathrm{~K}$; below they deviate upon cooling. The deviation may be attributed to short range AFM correlations for $\mathrm{T}<50 \mathrm{~K}$. Note that $\mathrm{Tb}$ behaves similarly below $50 \mathrm{~K}$ (Fig. S6e). This suggests that short-range AFM ordering is a general feature in the $i$-MAX series. Extracted information from the fitting gives an $\mu_{\text {eff }}$ of $8.34 \mu_{\mathrm{B}} / \mathrm{Gd}$, which is comparable to its free ion value of $7.94 \mu_{\mathrm{B}}$. A negative $\mathrm{C}$ W temperature, $\theta=-20.3 \mathrm{~K}$, supports the AFM correlations in this phase. Magnetization increases almost linearly and shows no sign of saturation up to 9T. Magnetization reaches $2.14 \mu_{\mathrm{B}} / \mathrm{Gd}$ at 9T.

The M/H vs. T curve for Gd (left y-axis in Fig. 5b) displays a small bump at $\mathrm{T}_{\mathrm{N}}=26 \mathrm{~K}$, which neither shows a temperature hysteresis nor a split between the zero field cooling and field cooling curves. Moreover, the bump does not shift with increasing $\mathrm{H}$, it just gets more pronounced (Fig. S7a). To link the observed bump to changes in electronic structure, we measured the temperature dependence of the electrical resistance, R. The results are shown in Fig. S7b. From 300 $\mathrm{K}$ to $50 \mathrm{~K}$ the response is metallic-like in that the resistance decreases linearly with decreasing temperatures. From 50 $\mathrm{K}$ to $26 \mathrm{~K}, \mathrm{R}$ (plotted on right $\mathrm{y}$-axis in Fig. $\mathbf{5 b}$ as a ratio to the $\mathrm{R}$ at $300 \mathrm{~K}$ ) levels off. Below $26 \mathrm{~K}$, R decreases linearly again until $5 \mathrm{~K}$. The sharp deflection on the R(T) curve at 26 K supports a distinct magnetic transition. The latter can also be seen from Arrott plots above and below $\mathrm{T}_{\mathrm{N}}$, respectively. Above $\mathrm{T}_{\mathrm{N}}$, the Arrott plots are linear (Fig. S7c). Below $\mathrm{T}_{\mathrm{N}}$, (Fig. S7d) inflections can be seen at $15 \mathrm{~K}$ and $20 \mathrm{~K}$. Overall, we conclude that the $\mathrm{Gd}$ phase crosses a magnetic transition at a $\mathrm{T}_{\mathrm{N}}$ of $26 \mathrm{~K}$.
(Mo2/3 Tb 1/3) 2 AlC: The functional dependence of M/H vs. $\mathrm{T}$ curves on $\mathrm{H}$ are shown in Fig. 6a (left y-axis). Below $1 \mathrm{~T}$, a sharp AFM transition at $20 \mathrm{~K}$ - denoted as $\mathrm{T}_{\mathrm{N} 1}$ - is observed. The AFM ordering is also supported by $\mathrm{M}(\mathrm{H})$ curves which show field induced transitions, marked with arrows, at $2 \mathrm{~K}$ (Fig. 6c). Here another faint anomaly can be seen at $28 \mathrm{~K}$ henceforth referred to as $\mathrm{T}_{\mathrm{N} 2}$, marked with an arrow in Fig. 6a. The temperature dependence of $c_{p}$ (right $y$-axis in Fig. 6a), clearly shows two lambda anomalies at $\mathrm{T}_{\mathrm{N} 1}$ and $\mathrm{T}_{\mathrm{N} 2}$ in zero field. Field dependent $\mathrm{c}_{\mathrm{p}}$ measurements (Fig. 6b) show that the anomaly at $\mathrm{T}_{\mathrm{N} 1}$ shifts towards lower- $\mathrm{T}$ with increasing $\mathrm{H}$, and disappears above $5 \mathrm{~T}$. The anomaly at $\mathrm{T}_{\mathrm{N} 2}$ also shifts progressively to lower $\mathrm{T}$, however, as opposed to the $\mathrm{T}_{\mathrm{N} 1}, \mathrm{~T}_{\mathrm{N} 2}$ is rather robust up to $9 \mathrm{~T}$.

To evaluate the CEF effect, we evaluated the magnetic entropy, $S_{M}$, by subtracting $c_{p}$ of the nonmagnetic Lu compound from $c_{p}$ of the Tb phase (see Fig. S8a). The estimated $\mathrm{S}_{\mathrm{M}}$ is shown in Fig. S8b and reaches $\mathrm{R} \ln 2$ around $24 \mathrm{~K}$ where $\mathrm{R}$ is ideal gas constant. The $\mathrm{Tb}^{3+}$ ion is a non-Kramers ion and CEF interactions lift the degeneracy of the $\mathrm{J}=6$ ground state multiplets into singlets. The value of $\mathrm{S}_{M}$ suggests that the first excited level is quite close to the ground state.

The $\mathrm{M}$ vs. $\mathrm{H}$ curves for the $\mathrm{Tb}$ phase in the 2 to $50 \mathrm{~K}$ temperature range (Fig. S9a) show three field-induced transitions, at $\sim 1 \mathrm{~T}, \sim 6.1 \mathrm{~T}$ and $\sim 7.1 \mathrm{~T}$ at $2 \mathrm{~K}$. These transitions are more easily seen in a dM/dH plot (Fig. S9b). Under $9 \mathrm{~T}$, the $\mathrm{M}$ reaches $3.4 \mu_{\mathrm{B}} / \mathrm{Tb}$, a value about one third that of the $9 \mu \mathrm{B}$ of the saturated $\mathrm{Tb}$ moment.

To investigate the magnetic structure, we performed NPD at 8,22 and $100 \mathrm{~K}$. Figures $\mathbf{6 d}$ and e show the magnetic scattering obtained by subtracting the pattern at $100 \mathrm{~K}$ (Fig. $1 j)$. Below $\mathrm{T}_{\mathrm{N} 1}$, additional magnetic peaks appear. These peaks can be indexed using the propagation vector $\mathbf{k}=(0$, $1 / 2,0)$. After checking all possible magnetic models - described in detail in section $\mathbf{S 6}$ - the best refinement with magnetic $R_{\mathrm{MAG}}=7.2 \%$ was obtained by the $1^{\text {st }}, 2^{\text {nd }}$, and $3^{\text {rd }}$ basis vectors of irreducible representation $\Gamma_{2}$. The refined magnetic moment of half of the Tb at $8 \mathrm{~K}$ is $4.9(2) \mu_{\text {в. The }}$ other half of the $\mathrm{Tb}$ atoms is nonmagnetic. A schematic of the alternating plane of magnetic and nonmagnetic $\mathrm{Tb}$ is shown in Fig. 6 f (upper). At $22 \mathrm{~K}$, between $\mathrm{T}_{\mathrm{N} 1}$ and $\mathrm{T}_{\mathrm{N} 2}$, another set of additional peaks appears. The new Bragg peaks are inconsistent with any commensurate magnetic structure. The propagation vector that gives the best agreement is $\mathbf{k}=(0,0.605,0)$. The best solution was obtained by considering the $1^{\text {st }}, 3^{\text {rd }}$, and $4^{\text {th }}$ basis vectors of the irreducible representation $\Gamma_{2}$, with $R_{\mathrm{MAG}}=12.16 \%$. The solution with $1^{\text {st }}$, $3^{\text {rd }}$, and $6^{\text {th }}$ basis vectors gives comparable agreement to the observed NPD, with $R_{\mathrm{MAG}}=12.20 \%$. The magnetic model corresponds to a sinewave-like structure. The refined magnetic moment oscillates from $0 \mu_{\mathrm{B}}$ up to $5.0(2) \mu_{\mathrm{B}}$. A schematic is shown in Fig. 6f (lower).

The apparent "nonmagnetic" state of every second $\mathrm{Tb}$ atom follows the sinewave modulation for this structure. These particular $\mathrm{Tb}$ atoms are positioned in the nodes of that modulation causing their magnetic moments to cancel. This does not happen for the incommensurate $\mathbf{k}=(0,0.605$, $0)$ structure by geometrical considerations.

From the results shown in Fig. S6e, a small, negative $\theta=-$ 12.1 $\mathrm{K}$ and $\mu_{\text {eff }}=9.78(3) \mu_{\mathrm{B}}$ are obtained for the $\mathrm{Tb}$ phase. The $\mathrm{M}$ is not saturated at $9 \mathrm{~T}$, and reaches only $3.4 \mu_{\mathrm{B}}$. We 
would expect a saturated moment of $\sim 5 \mu_{\mathrm{B}}$. The reduction of magnetic moment is likely due to CEF interaction ${ }^{35}$.

$\left(\mathbf{M o}_{2 / 3} \mathbf{E r}_{1 / 3}\right)_{2}$ AlC: A plot of M/H vs. T for this phase, at $\mathrm{H}$ $=0.01 \mathrm{~T}$, is shown by a solid line in Fig. 7a (left y-axis). A magnetic transition is barely visible. A pronounced lambda anomaly in $c_{p}$ is observed at $\mathrm{T}_{\mathrm{N}}=3.6 \mathrm{~K}$ (right $\mathrm{y}$-axis in Fig. 7a). The M vs. H curves (Fig. 7b) show no hysteresis and no remanence. The magnetization approaches $\sim 6 \mu_{\text {в }} /$ Er at 9 T. At $2 \mathrm{~K}$ (black line in Fig. 7b), a kink can be seen at $\sim 1 \mathrm{~T}$, implying a field induced transition. Fig. S6g shows the fitting of $\mathrm{H} / \mathrm{M}$ vs. $\mathrm{T}$ to the Curie-Weiss law resulting in 9.24(1) $\mu_{\text {в }}$ per Er atom, a value that is close to the free ion effective value.

To shed light on the crystal and magnetic structures of this phase, we performed NPD at different temperatures. When the NPD data at $298 \mathrm{~K}$ (not shown) and $22 \mathrm{~K}$ (Fig. 7c) were compared, no evidence for any structural transitions was seen. The LPs of all three axes decreased upon cooling. The unit cell volume contracts by $0.45 \%$, which is typical of the $i$-MAX phases ${ }^{36}$. Upon cooling below $3.6 \mathrm{~K}$, however, the phase magnetically orders, and new reflections emerge, which do not coincide with the crystal structure. The magnetic scattering spectrum of the Er phase at $1.5 \mathrm{~K}$ (Fig. 7d) is obtained by subtracting the $22 \mathrm{~K}$ profile (Fig. 7c).

Attempts to fit the diffraction pattern at $1.5 \mathrm{~K}$ using propagation vectors that belong to special symmetry points in the Brillouin zone resulted in a propagation vector $\mathbf{k}_{1}=$ $(1 / 2,0,1 / 2)$ with which part of the magnetic structure was refined. To fit the remaining magnetic structure, a systematic search of $\mathrm{k}$ along lines within the Brillouin zone, combined with a Monte Carlo refinement of the spins configuration at each $k$, was performed using the SARAh code ${ }^{37,38}$. The incommensurate propagation vector $\mathbf{k}_{2}=(0,0.675(1)$, 0 ), was found to successfully index the remaining magnetic reflections. The details are described in Section S6. The ordered magnetic moments corresponding to the $\mathbf{k}_{\mathbf{1}}$, and $\mathbf{k}_{\mathbf{2}}$ structures were refined to be $1.00(3)\left(R_{\text {mag }}=11.2 \%\right)$, and $5.6(2) \mu_{\mathrm{B}} / \operatorname{Er}\left(R_{\operatorname{mag}}=10.3\right)$, respectively. Importantly, like in the $\mathrm{Tb}$ case, the Er ordered magnetic moment is significantly reduced compared to the $\mathrm{Er}^{3+}$ free-ion moment ( 9 $\left.\mu_{\mathrm{B}}\right)$, most probably due to similar considerations.

\section{Discussion and Conclusion}

In the Sixties, Nowotny et al. discovered - in powder form - roughly 50 MAX phases. The original set of M elements was $\mathrm{Ti}, \mathrm{Nb}, \mathrm{Zr}, \mathrm{Hf}, \mathrm{V}, \mathrm{Mo}, \mathrm{Ta}$, and Cr. These phases were then mostly ignored for the next two decades. Interest in these phases was revitalized in the mid-Nineties ${ }^{39}$ and has since remained high ${ }^{12}$. In 2013 we discovered the first magnetic MAX phase and added Mn to the original set of M elements ${ }^{40}$. In 2017, we discovered the $i$-MAX phases, which added Sc, $\mathrm{Y}$, and $\mathrm{W}$ to the mix ${ }^{16-18}$. The only study on a RE MAX phase was on the superconductivity of $\mathrm{Lu}_{2} \mathrm{SnC}^{41}$. Herein, we add eleven new elements and essentially double the known MAX-phase M-elements by realizing a new class of RE-based magnetic $i$-MAX phases.

These phases display a wide range of magnetic characteristics, ranging from incommensurate magnetic structures, magnetization plateaus, to multi- $k$ structures, etc. We suggest that this behavioral variety is a result of competing long ranged exchange interactions in the presence of magnetocrystalline anisotropy. Various non-collinear AFM states are found in these phases, in particular for the Tb and Er phases.
Recently, reports on 2D magnetic materials (monolayers of $\mathrm{Cr}_{2} \mathrm{Ge}_{2} \mathrm{Te}_{6}$ and $\mathrm{CrI}_{3}$ ) have appeared in the literature, displaying layer-dependent magnetic phenomena ${ }^{45,46}$. Close to all MXenes (the 2D derivative of the MAX phases) to date originate from selective etching of the $\mathrm{Al}$ layer from a MAX phase ${ }^{47}$. This concept was recently expanded by selectively etching both $\mathrm{Al}$ and $\mathrm{Sc}$ from $\left(\mathrm{Mo}_{2 / 3} \mathrm{Sc}_{1 / 3}\right)_{2} \mathrm{AlC} i$-MAX, resulting in a new 2D solid with ordered divacancies ${ }^{16}$. Furthermore, "targeted etching" was proposed for the (Mo2/3 $\left.\mathrm{Y}_{1 / 3}\right)_{2} \mathrm{AlC} i$-MAX, showing evidence for etching procedures resulting in vacancy formation or the realization of chemically ordered MXenes based on Mo and $\mathrm{Y}^{48}$. All novel $i$-MAX phases presented herein are Al based. Therefore, at least in principle, it should be straightforward to convert them to their 2D counterparts, which is ongoing.

Herein we report on the synthesis of a MAX-phase based, RE-containing family of ordered, layered solids, with complex inter- and intraplanar magnetic order. The chemistry of this family is $\left(\mathrm{Mo}_{2 / 3} \mathrm{RE}_{1 / 3}\right)_{2} \mathrm{AlC}$, with the $\mathrm{RE}$ atoms positioned at the apexes of a 2D triangular lattice. Eleven phases were discovered for RE $=\mathrm{Ce}, \mathrm{Pr}, \mathrm{Nd}, \mathrm{Sm}, \mathrm{Gd}, \mathrm{Tb}, \mathrm{Dy}, \mathrm{Ho}, \mathrm{Er}$, $\mathrm{Tm}$, and Lu, which crystalize in three polymorphs with $C 2 / c$, $\mathrm{C} 2 / \mathrm{m}$, and $\mathrm{Cmcm}$ symmetries. The 2D metal carbide blocks are identical. We thus label this family, RE $i$-MAX. Using Tb and Er quaternaries as examples we demonstrate the complex magnetic order - characteristic of magnetically frustrated systems due to competing intra- and in-plane magnetic interactions. Both structures contain incommensurate as well as commensurate magnetic modulations depending on temperature. Interestingly, the incommensurate structures of the $\mathrm{Tb}$ and Er compounds are similar, while the commensurate ones differ albeit them sharing the same crystallographic symmetry. This suggests strong dependency of magnetic interactions on the RE atoms as well. The prospect that some of these phases may be converted to their 2D counterparts ${ }^{48}$ adds to their potential importance.

\section{ASSOCIATED CONTENT}

Supporting Information.

Section S1. Crystal structure from STEM and XRD.

Section S2. Relationship between the three polymorphs.

Section S3. Theoretical modeling.

Section S4. Curie-Weiss fitting in the paramagnetic state

Section S5. Magnetism of $\left(\mathrm{Mo}_{2 / 3} \mathrm{Gd}_{1 / 3}\right)_{2} \mathrm{AlC}$ (Mo $\left.2 / 3 \mathrm{~Tb}_{1 / 3}\right)_{2} \mathrm{AlC}$

Section S6. Detailed magnetic structure analysis of the NPD data

Section S7. Structural parameters from NPD.

Fig. S1. Crystal structure of REMAX.

Fig. S2. Rietveld refinement of XRD.

Fig. S3. Schematic illustration of different stacking sequences for REMAX.

Fig. S4. Schematic representation of six collinear spin configurations.

Fig. S5. Local magnetic moment of RE.

Fig. S6. H/M vs. T and fitting to the Curie-Weiss law.

Fig. S7. Magnetic behavior of $\left(\mathrm{Mo}_{2 / 3} \mathrm{Gd}_{1 / 3}\right)_{2} \mathrm{AlC}$.

Fig. S8. Heat capacity and magnetic entropy.

Fig. S9. Magnetism of $\left(\mathrm{Mo}_{2 / 3} \mathrm{~Tb}_{1 / 3}\right)_{2} \mathrm{AlC}$.

Table S1. Cell parameters and atom coordinates obtained from XRD Rietveld refinement

Table S2. Phase purity from Rietveld refinement.

Table S3. Structural refinement from NPD. 
This material is available free of charge via the Internet at http://pubs.acs.org.

\section{AUTHOR INFORMATION}

\section{Corresponding Author}

* to whom correspondence should be addressed quanzheng.tao@liu.se or johanna.rosen@liu.se

\section{Author Contributions}

J.R. initiated and supervised the study. Q.T., A.P., and R.M. conducted the synthesis. Q.T and E.N.C. performed XRD analysis. L.H. oversaw the MAX phase STEM analysis performed by J.L. M.D. performed the ab initio calculations, with B. J. providing guidance. Q.T., A.M., S.C., O.R., D. P, E.N.C., H.S., A.H., C.R., and A.W. contributed to the neutron experiment and analysis. Q.T., C.O., R.G., R.S., U.W., and M.F. contributed to the magnetic characterizations. Q.T., M.F., M.W.B., and J.R. wrote the manuscript with contributions from the other coauthors. All co-authors read and commented on successive drafts of the manuscript.

\section{ACKNOWLEDGMENT}

J.R., P.P, and L.H. acknowledge support from the Knut and Alice Wallenberg (KAW) Foundation for a Scholar Grant, a Fellowship Grant, Project funding (KAW 2015.0043), and for support to the Linköping Ultra Electron Microscopy Laboratory. The Swedish Research Council is gratefully acknowledged through Project 642-2013-8020, 2015-00607, and 621-2014-4890. The calculations were carried out using supercomputer resources provided by the Swedish National Infrastructure for Computing (SNIC) at the National Supercomputer Centre (NSC), the High Performance Computing Center North (HPC2N), and the PDC Center for High Performance Computing. J.R. and P.P. also acknowledge the Swedish Government Strategic Research Area in Materials Science on Functional Materials at Linköping University (Faculty Grant SFO-Mat-LiU No. 2009-00971). R.S. acknowledges DFG funding under Grant SA 3095/2-1. A portion of this research used resources at the High Flux Isotope Reactor, a DOE Office of Science User Facility operated by the Oak Ridge National Laboratory. We thank HZB for the allocation of neutron radiation beamtime. D.P, O.R., and E.N.C acknowledge the support of the IAEC Pazy foundation grant.

\section{REFERENCES}

1. Riseborough, P. S., Heavy fermion semiconductors. Adv. Phys. 2000, 49 (3), 257-320.

2. Dzero, M.; Xia, J.; Galitski, V.; Coleman, P., Topological Kondo insulators. Annu. Rev. Condensed Matter. Phys. 2016, 7, 249-280.

3. Xu, Y.; Yue, C.; Weng, H.; Dai, X., Heavy Weyl Fermion State in CeRu 4 Sn 6. Phys. Rev. X 2017, 7 (1), 011027.

4. Brando, M.; Belitz, D.; Grosche, F.; Kirkpatrick, T., Metallic quantum ferromagnets. Rev. Mod. Phys. 2016, 88 (2), 025006.

5. Wirth, S.; Steglich, F., Exploring heavy fermions from macroscopic to microscopic length scales. Nat. Rev. Mater. 2016, 1, 16051.

6. Tsujimoto, M.; Matsumoto, Y.; Tomita, T.; Sakai, A.; Nakatsuji, S., Heavy-fermion superconductivity in the quadrupole ordered state of $\operatorname{PrV}_{2} \mathrm{Al}_{20}$. Phys. Rev. lett. 2014, 113 (26), 267001.

7. Akintola, K.; Pal, A.; Potma, M.; Saha, S.; Wang, X.; Paglione, J.; Sonier, J., Quantum spin fluctuations in the bulk insulating state of pure and Fe-doped SmB 6. Phys. Rev. B 2017, 95 (24), 245107.

8. Sanada, S.; Aoki, Y.; Aoki, H.; Tsuchiya, A.; Kikuchi, D.; Sugawara, H.; Sato, H., Exotic heavy-fermion state in filled Skutterudite $\mathrm{SmOs}_{4} \mathrm{Sb}_{12}$. J. Phys. Soc. Jpn. 2005, 74 (1), 246-249.
9. Lu, F.; Zhao, J.; Weng, H.; Fang, Z.; Dai, X., Correlated topological insulators with mixed valence. Phys. Rev. Lett. 2013, 110 (9), 096401.

10. Barsoum, M. W., The $M_{N+1} A_{N}$ phases: A new class of solids: Thermodynamically stable nanolaminates. Prog. Solid State Chem. 2000, 28 (1-4), 201-281.

11. Barsoum, M. W.; Radovic, M., Elastic and mechanical properties of the MAX phases. Annu. Rev. Mater. Res. 2011, 41, 195-227.

12. Barsoum, M. W., MAX phases: properties of machinable ternary carbides and nitrides. John Wiley \& Sons: 2013.

13. Fashandi, H.; Dahlqvist, M.; Lu, J.; Palisaitis, J.; Simak, S. I.; Abrikosov, I. A.; Rosen, J.; Hultman, L.; Andersson, M.; Spetz, A. L., Synthesis of $\mathrm{Ti}_{3} \mathrm{AuC}_{2}, \mathrm{Ti}_{3} \mathrm{Au}_{2} \mathrm{C}_{2}$ and $\mathrm{Ti}_{3} \mathrm{IrC}_{2}$ by noble metal substitution reaction in $\mathrm{Ti}_{3} \mathrm{SiC}_{2}$ for high-temperature-stable Ohmic contacts to SiC. Nat. Mater. 2017, 16, 814-818.

14. Naguib, M.; Kurtoglu, M.; Presser, V.; Lu, J.; Niu, J.; Heon, M.; Hultman, L.; Gogotsi, Y.; Barsoum, M. W., Two-dimensional nanocrystals produced by exfoliation of $\mathrm{Ti}_{3} \mathrm{AlC}_{2}$. Adv. Mater. 2011, 23 (37), 4248-4253.

15. Anasori, B.; Lukatskaya, M. R.; Gogotsi, Y., 2D metal carbides and nitrides (MXenes) for energy storage. Nat. Rev. Mater. 2017, 2 (2), 16098.

16. Tao, Q.; Dahlqvist, M.; Lu, J.; Kota, S.; Meshkian, R.; Halim, J.; Palisaitis, J.; Hultman, L.; Barsoum, M. W.; Persson, P. O., Two-dimensional Mo ${ }_{1.33} \mathrm{C}$ MXene with divacancy ordering prepared from parent 3D laminate with in-plane chemical ordering. Nat. Comm. 2017, 8, 14949.

17. Dahlqvist, M.; Lu, J.; Meshkian, R.; Tao, Q.; Hultman, L.; Rosen, J., Prediction and synthesis of a family of atomic laminate phases with Kagomé-like and in-plane chemical ordering. Sci. Adv. 2017, 3 (7), e1700642.

18. Meshkian, R.; Dahlqvist, M.; Lu, J.; Wickman, B.; Halim, J.; Thörnberg, J.; Tao, Q.; Li, S.; Intikhab, S.; Snyder, J.; Barsoum, M. W.; Yildizhan, M.; Palisaitis, J.; Hultman, L.; Persson, P. O. Å.; Rosen, J., WBased Atomic Laminates and Their 2D Derivative $\mathrm{W}_{1.33} \mathrm{C}$ MXene with Vacancy Ordering. Adv. Mater. 2018, 30 (21), 1706409.

19. Ye, L.; Kang, M.; Liu, J.; von Cube, F.; Wicker, C. R.; Suzuki, T.; Jozwiak, C.; Bostwick, A.; Rotenberg, E.; Bell, D. C., Massive Dirac fermions in a ferromagnetic kagome metal. Nature 2018, 555, 638642.

20. Balents, L., Spin liquids in frustrated magnets. Nature 2010, 464 (7286), 199.

21. Guo, H.-M.; Franz, M., Topological insulator on the kagome lattice. Phys. Rev. B 2009, 80 (11), 113102.

22. Xu, G.; Lian, B.; Zhang, S.-C., Intrinsic Quantum Anomalous Hall Effect in the Kagome Lattice $\mathrm{Cs}_{2} \mathrm{LiMn}_{3} \mathrm{~F}_{12}$. Phys. Rev. Lett. 2015, 115 (18), 186802.

23. Kresse, G.; Hafner, J., Ab initio molecular dynamics for liquid metals. Phys. Rev. B 1993, 47 (1), 558-561.

24. Kresse, G.; Furthmüller, J., Efficiency of ab-initio total energy calculations for metals and semiconductors using a plane-wave basis set. Compu. Mater. Sci. 1996, 6 (1), 15-50.

25. Kresse, G.; Furthmüller, J., Efficient iterative schemes for ab initio total-energy calculations using a plane-wave basis set. Phys. Rev. B 1996, 54 (16), 11169-11186.

26. Blöchl, P. E., Projector augmented-wave method. Phys. Rev. B 1994, 50 (24), 17953-17979.

27. Kresse, G.; Joubert, D., From ultrasoft pseudopotentials to the projector augmented-wave method. Phys. Rev. B 1999, 59 (3), 1758-1775.

28. Perdew, J. P.; Burke, K.; Ernzerhof, M., Generalized gradient approximation made simple. Phys. Rev. Lett. 1996, 77 (18), 38653868.

29. Monkhorst, H. J.; Pack, J. D., Special points for Brillouin-zone integrations. Phys. Rev. B 1976, 13 (12), 5188-5192. 
30. Dudarev, S. L.; Botton, G. A.; Savrasov, S. Y.; Humphreys, C. J.; Sutton, A. P., Electron-energy-loss spectra and the structural stability of nickel oxide: An LSDA+U study. Phys. Rev. B 1998, 57 (3), 1505-1509.

31. Gschneidner, K. A., Physical properties of the rare earth metals. Bull. Alloy Phase Diagrams 1990, 11 (3), 216-224.

32. Hughes, I.; Däne, M.; Ernst, A.; Hergert, W.; Lüders, M.; Poulter, J.; Staunton, J.; Svane, A.; Szotek, Z.; Temmerman, W., Lanthanide contraction and magnetism in the heavy rare earth elements. $\mathrm{Na}$ ture 2007, 446 (7136), 650-653.

33. Farle, M.; Lewis, W.; Baberschke, K., Detailed analysis of the in situ magneto-optic Kerr signal of gadolinium films near the Curie temperature. Appl. Phys. Lett. 1993, 62 (21), 2728-2730.

34. Opagiste, C.; Jackson, M.; Galéra, R.-M.; Lhotel, E.; Paulsen, C.; Ouladdiaf, B., Metamagnetic behaviour of Nd3Pt23Si11. J. Mag. Mag. Mater. 2013, 340, 46-49.

35. Rivin, O.; Osborn, R.; Kolesnikov, A. I.; El'ad, N. C.; Shaked, H., $\mathrm{Tb}^{3+}$ in $\mathrm{TbCo}_{3} \mathrm{~B}_{2}$ : A singlet ground state system studied by inelastic neutron scattering. Phys. Rev. B 2008, 78 (18), 184424.

36. Caspi, E. a. N.; Chartier, P.; Porcher, F.; Damay, F.; Cabioc'h, T., Ordering of $(\mathrm{Cr}, \mathrm{V})$ Layers in Nanolamellar $\left(\mathrm{Cr}_{0.5} \mathrm{~V}_{0.5}\right)_{\mathrm{n}+1} \mathrm{AlC}_{\mathrm{n}} \mathrm{Com}-$ pounds. Mater. Res. Lett. 2015, 3 (2), 100-106.

37. Wills, A., A new protocol for the determination of magnetic structures using simulated annealing and representational analysis (SARAh). Physica B: Condensed Matter. 2000, 276, 680-681.

38. Wills, A., Indexing magnetic structures and crystallographic distortions from powder diffraction: Brillouin zone indexing. Z. Kristallogr. 2009, 30, 39-44.

39. Barsoum, M. W.; El-Raghy, T., Synthesis and characterization of a remarkable ceramic: $\mathrm{Ti}_{3} \mathrm{SiC}_{2}$. J. Am. Ceram. Soc. 1996, 79 (7), 1953-1956.

40. Ingason, A. S.; Mockuté, A.; Dahlqvist, M.; Magnus, F.; Olafsson, S.; Arnalds, U. B.; Alling, B.; Abrikosov, I. A.; Hjörvarsson, B.;
Persson, P. Å., Magnetic self-organized atomic laminate from first principles and thin film synthesis. Phys. Rev. Lett. 2013, 110 (19), 195502.

41. Kuchida, S.; Muranaka, T.; Kawashima, K.; Inoue, K.; Yoshikawa, M.; Akimitsu, J., Superconductivity in $\mathrm{Lu}_{2} \mathrm{SnC}$. Physica C: superconductivity 2013, 494, 77-79.

42. Suzuki, T.; Chisnell, R.; Devarakonda, A.; Liu, Y.-T.; Feng, W.; Xiao, D.; Lynn, J. W.; Checkelsky, J., Large anomalous Hall effect in a half-Heusler antiferromagnet. Nat. Phys. 2016, 12 (12), 1119.

43. Kuroda, K.; Tomita, T.; Suzuki, M.-T.; Bareille, C.; Nugroho, A.; Goswami, P.; Ochi, M.; Ikhlas, M.; Nakayama, M.; Akebi, S., Evidence for magnetic Weyl fermions in a correlated metal. Nat. Mater. 2017, 16 (11), 1090.

44. Šmejkal, L.; Mokrousov, Y.; Yan, B.; MacDonald, A. H., Topological antiferromagnetic spintronics. Nat. Phys. 2018, 14, 242-251.

45. Huang, B.; Clark, G.; Navarro-Moratalla, E.; Klein, D. R.; Cheng, R.; Seyler, K. L.; Zhong, D.; Schmidgall, E.; McGuire, M. A.; Cobden, D. H., Layer-dependent ferromagnetism in a van der Waals crystal down to the monolayer limit. Nature 2017, 546 (7657), 270-273.

46. Gong, C.; Li, L.; Li, Z.; Ji, H.; Stern, A.; Xia, Y.; Cao, T.; Bao, W.; Wang, C.; Wang, Y., Discovery of intrinsic ferromagnetism in twodimensional van der Waals crystals. Nature 2017, 546, 265-269.

47. Naguib, M.; Mashtalir, O.; Carle, J.; Presser, V.; Lu, J.; Hultman, L.; Gogotsi, Y.; Barsoum, M. W., Two-dimensional transition metal carbides. ACS nano 2012, 6 (2), 1322-1331.

48. Persson, I.; Ghazaly, A. e.; Tao, Q.; Halim, J.; Kota, S.; Darakchieva, V.; Palisaitis, J.; Barsoum, M. W.; Rosen, J.; Persson, P. O. Å., Tailoring Structure, Composition, and Energy Storage Properties of MXenes from Selective Etching of In-Plane, Chemically Ordered MAX Phases. Small 14 (17), 1703676. 
a
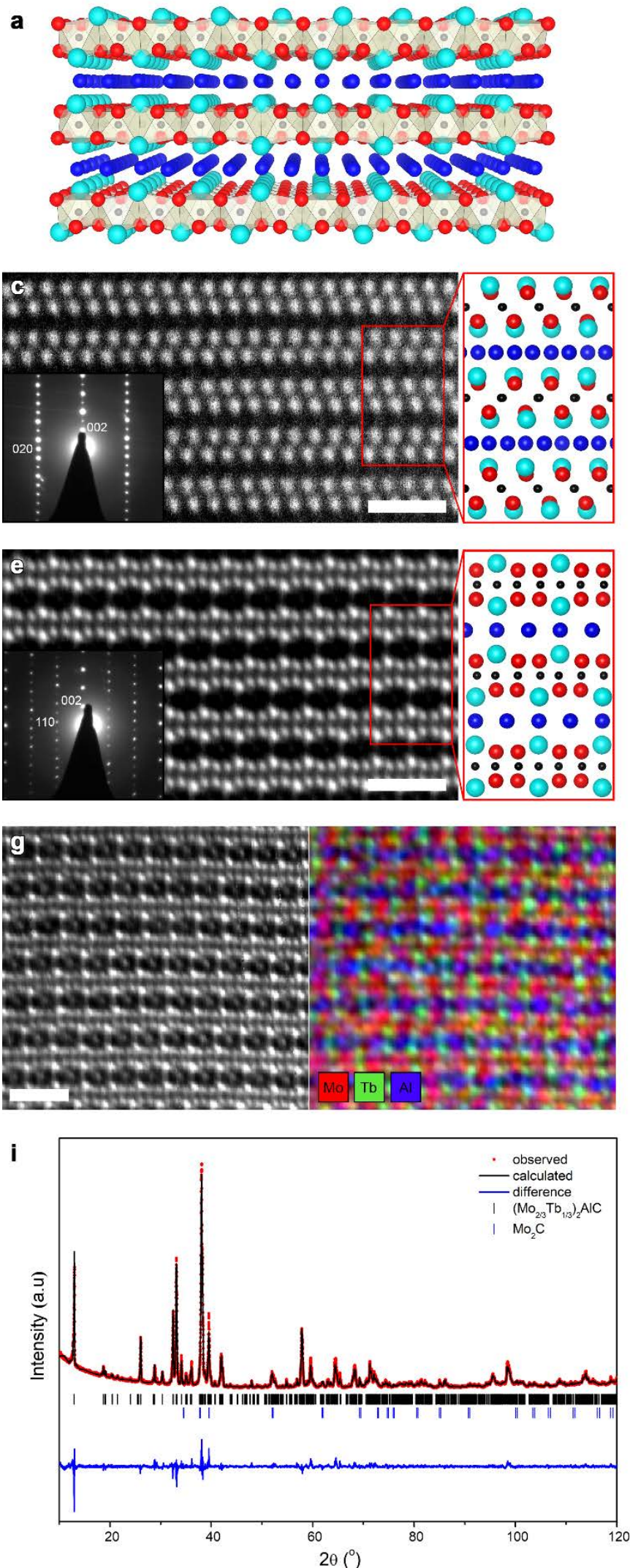

b
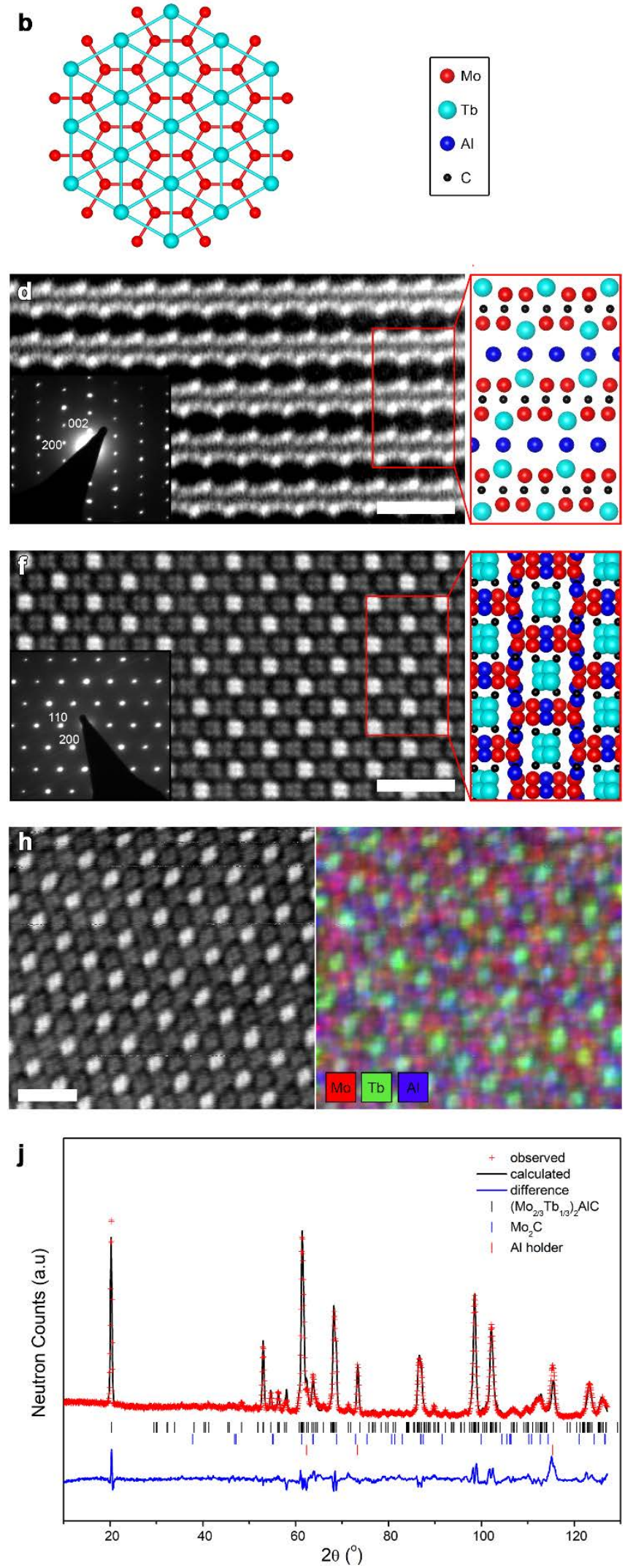

Fig. 1. Crystal structure of space group $C 2 / c$-MAX phase. (a) 3D perspective side view of the C2/c $i$-MAX structure with (b) corresponding top view of building block, (Mo2/3 RE $1 / 3)_{2} \mathrm{C}$ layer. STEM images of (Mo2/3 Tb1/3) 2 AlC along (c) [100], (d) [010], (e) [110], (f) [001] zone axis, respectively, with corresponding SAED. Schematics to the right of each panel represent the corresponding atomic arrangements assuming the structure is the space group $C 2 / c(15)$. Atomic resolution EDX mapping of ( $\left.\mathrm{Mo}_{2 / 3} \mathrm{~Tb}_{1 / 3}\right)_{2} \mathrm{AlC}$ along (g) [110] and (h) [100] zone axis. (i) Rietveld refinement of XRD pattern of $\left(\mathrm{Mo}_{2 / 3} \mathrm{~Tb}_{1 / 3}\right)_{2} \mathrm{AlC}$ at room temperature. (j) Rietveld refinement of NPD pattern of (Mo2/3Tb1/3)2AlC at $100 \mathrm{~K}$. Scale bars (c-h), $1 \mathrm{~nm}$. 

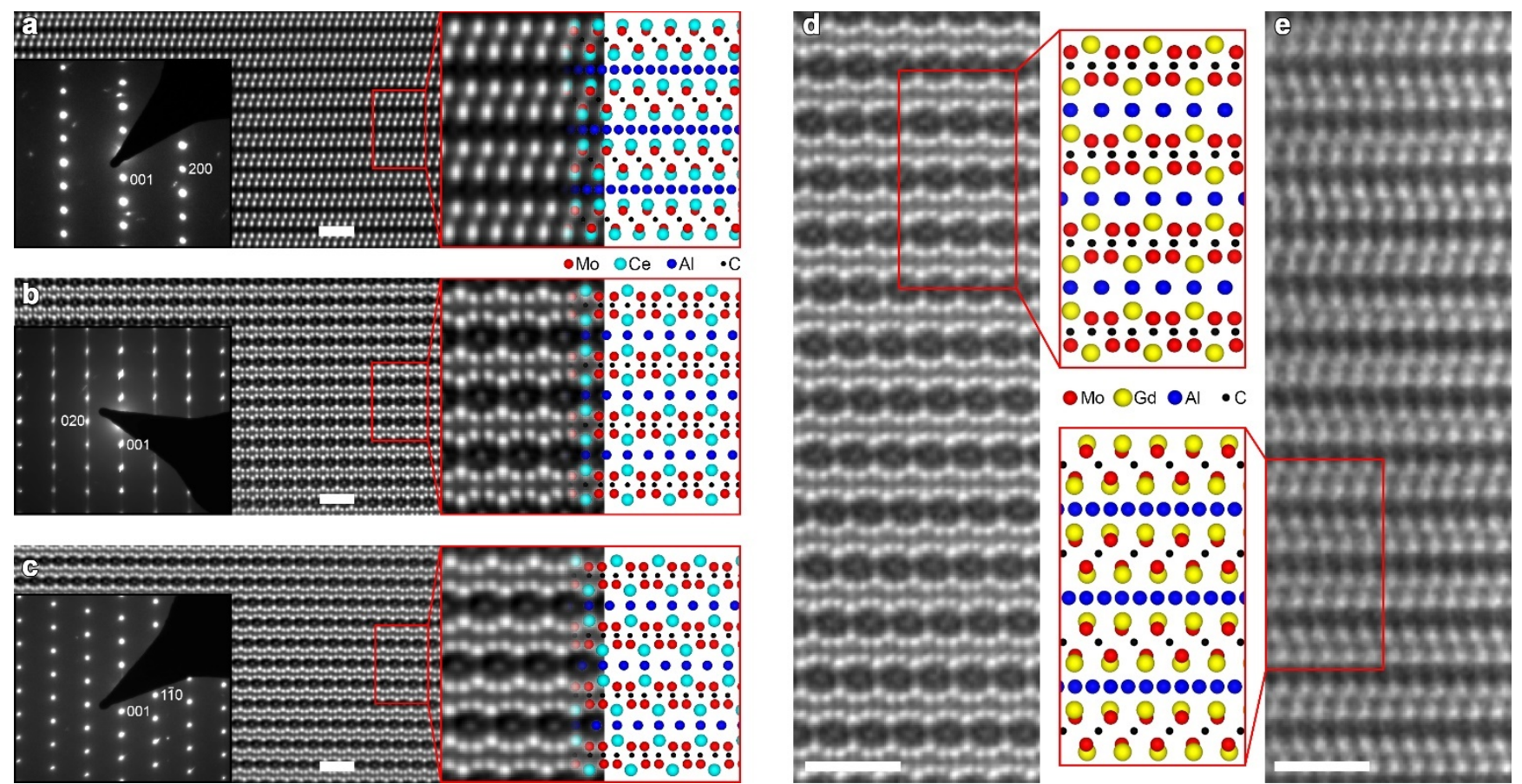

Fig. 2. Crystal structure of the $i$-MAX phases. STEM images of $\left(\mathrm{Mo}_{2 / 3} \mathrm{Ce}_{1 / 3}\right)_{2} \mathrm{AlC}$ along (a) [010], (b) [100], and (c) [110] zone axis, respectively, with corresponding SAED. Schematics to the right of each panel represent the corresponding atomic arrangements assuming the structure is the space group $C 2 / m(12)$. STEM images of $\left(\mathrm{Mo}_{2 / 3} \mathrm{Gd}_{1 / 3}\right)_{2} \mathrm{AlC}$ along (d) [110] and (e) [100] zone axis, respectively. Schematics to the right of each panel represent the corresponding atomic arrangements assuming the structure is the orthorhombic space group $\mathrm{Cmcm}$ (63). Scale bars, $1 \mathrm{~nm}$. 
a

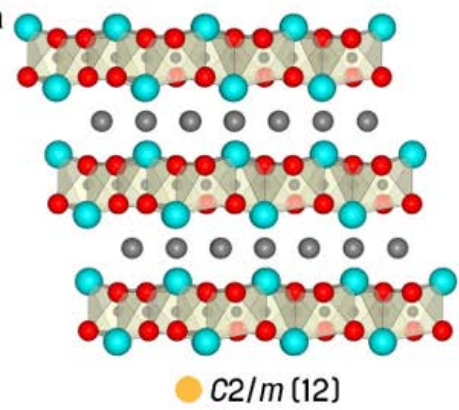

b

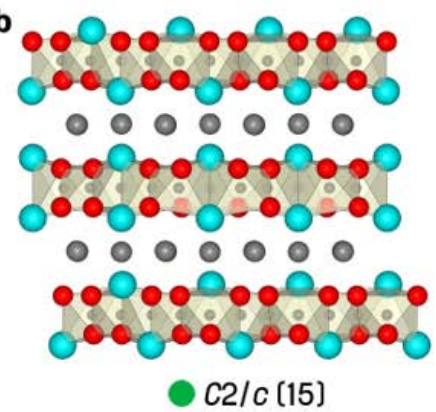

c

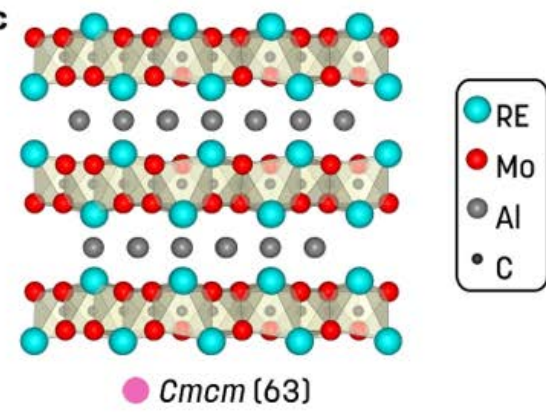

EU
$\mathrm{Yb}$
Lu

Fig. 3. Summary of crystal structures of the $i$-MAX phases. Schematic of $\left(\mathrm{Mo}_{2 / 3} \mathrm{RE}_{1 / 3}\right)_{2} \mathrm{AlC}$ crystallized in (a) monoclinic C2/m, (b) monoclinic $C 2 / c$, and (c) orthorhombic $\mathrm{Cmcm}$. (d) RE = Ce and Pr crystallize in $C 2 / m$, and RE = Nd, Tb, Dy, Ho, Er, Tm and Lu crystallize in $C 2 / c$. Two polymorphs are found for $\mathrm{RE}=\mathrm{Sm}$ and $\mathrm{Gd}$. 

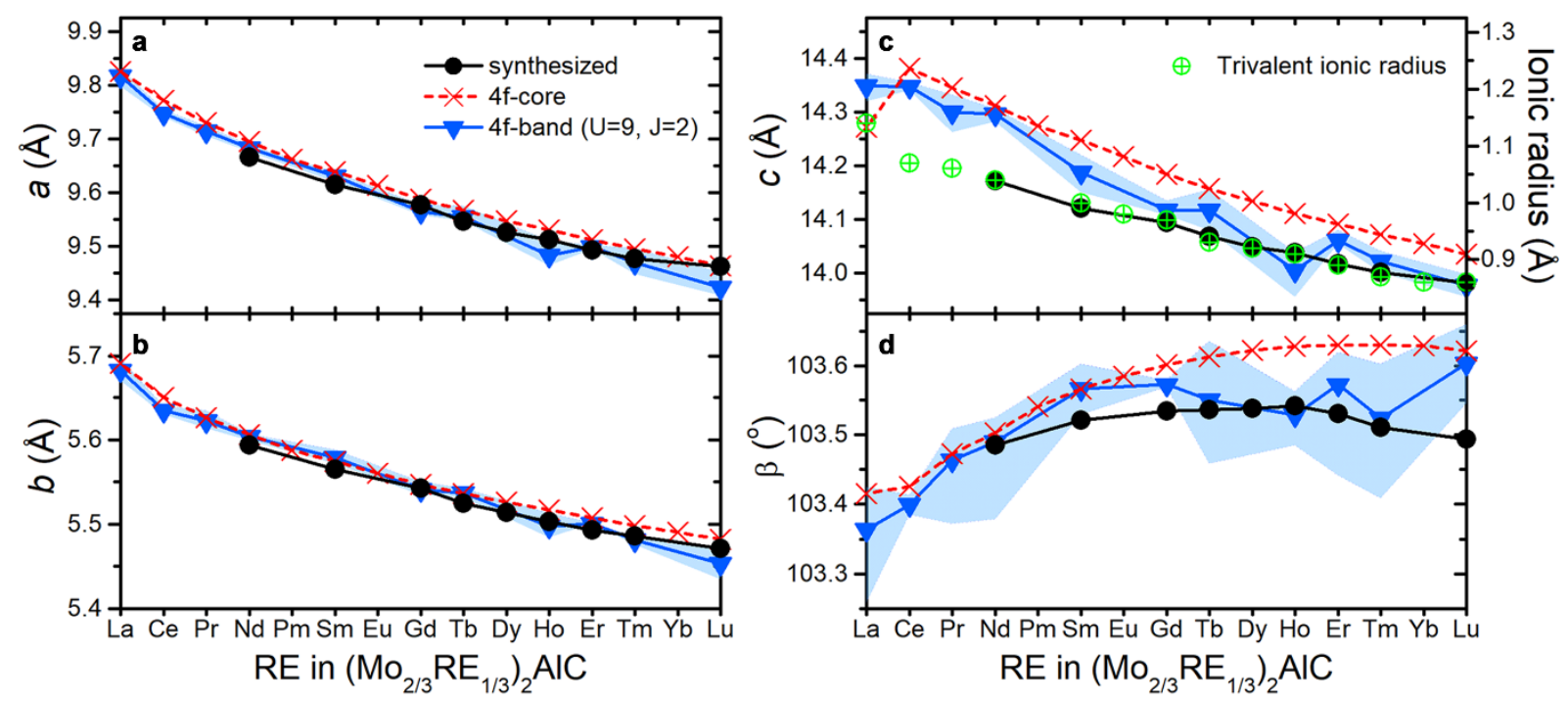

Fig. 4. Structural parameters and magnetic properties. Measured (XRD at RT) and calculated lattice parameters (a) $a$, (b) $b$, (c) $c$, and, (d) angle $\beta$ for $\left(\mathrm{Mo}_{2 / 3} \mathrm{RE}_{1 / 3}\right)_{2} \mathrm{AlC}$ crystallized in $C 2 / c$. Theoretical data are obtained by treating $4 \mathrm{f}$ electrons in core ( $4 f$-core) or as part of the valence band ( $4 \mathrm{f}$-band). For $4 f$-band we used $\mathrm{U}=9 \mathrm{eV}$ and $\mathrm{J}=2 \mathrm{eV}$. Data points for $4 f$-band are given as averaged value obtained for the lowest energy spin states, within $2 \mathrm{meV} /$ atom, and corresponding min and max values are represented by the blue shaded area. The ionic radius of trivalent RE is added in (c) for comparison. 

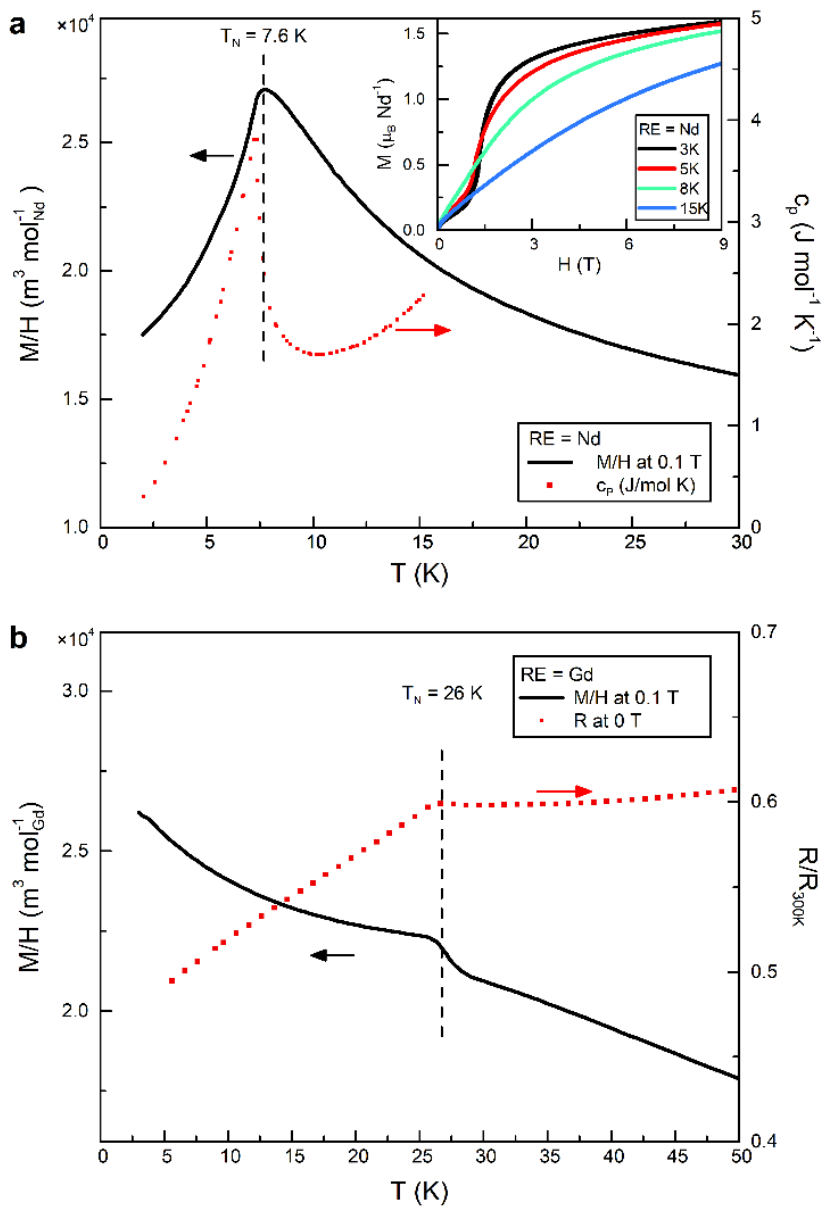

Figure 5. Magnetic properties. a. Magnetization and specific heat, $\mathrm{c}_{\mathrm{p}}$, vs. T for $\left(\mathrm{Mo}_{2 / 3} \mathrm{Nd}_{1 / 3}\right)_{2} \mathrm{AlC}$. b. Magnetization and resistivity vs $\mathrm{T}$ for $\left(\mathrm{Mo}_{2 / 3} \mathrm{Gd}_{1 / 3}\right)_{2} \mathrm{AlC}$. 

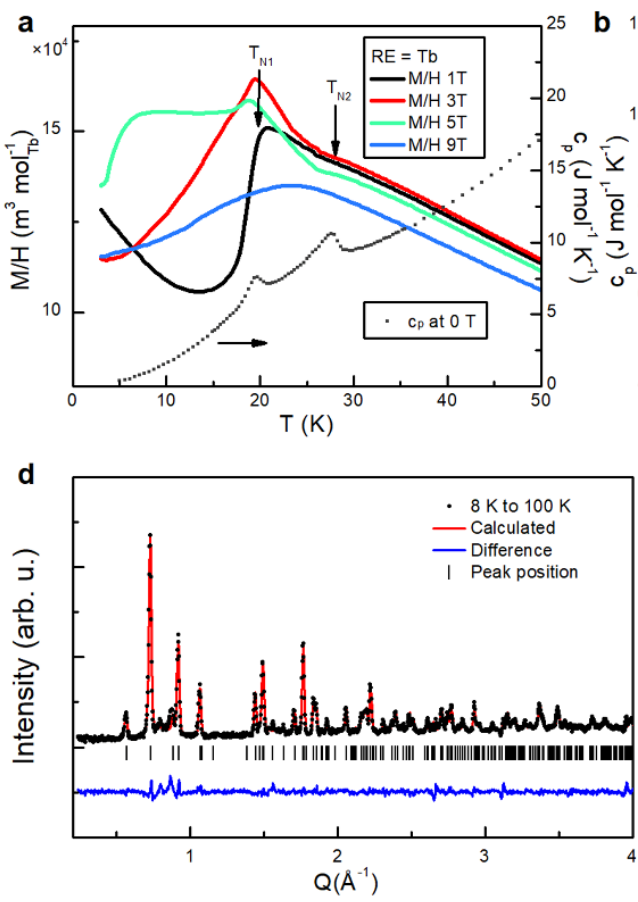
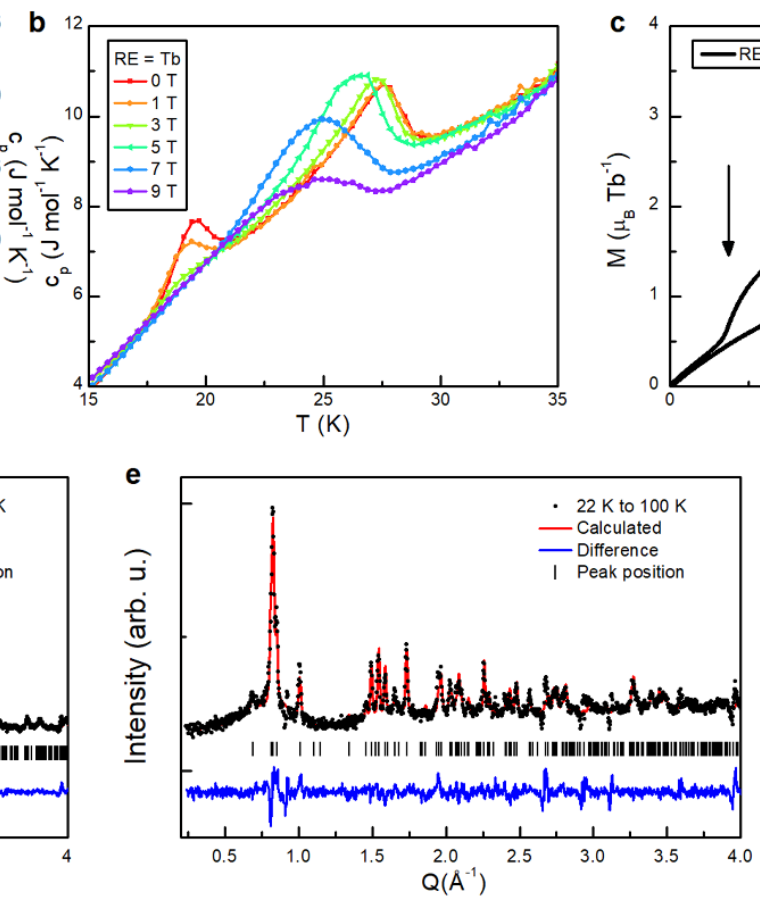

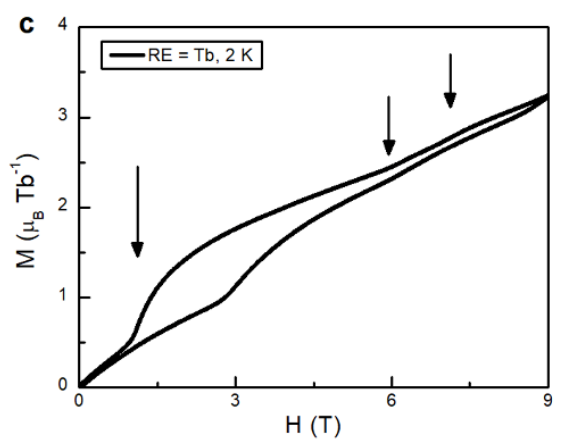

$\mathrm{H}(\mathrm{T})$

f

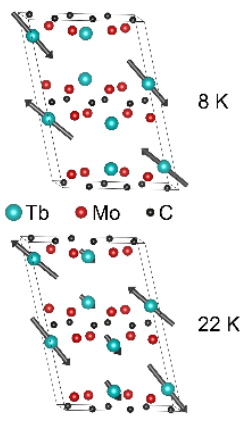

Fig. 6. Magnetic properties of $\left(\mathrm{Mo}_{2 / 3} \mathrm{~Tb}_{1 / 3}\right)_{2} \mathrm{AlC}$. a. M/H vs. T and $\mathrm{c}_{\mathrm{p}}$ vs. T at $0 \mathrm{~T}$ for $\left(\mathrm{Mo}_{2 / 3} \mathrm{~Tb}_{1 / 3}\right)_{2} \mathrm{AlC}$. b. $\mathrm{c}_{\mathrm{p}}$ vs. T at different magnetic fields for $\left(\mathrm{Mo}_{2 / 3} \mathrm{~Tb}_{1 / 3}\right)_{2} \mathrm{AlC}$. c. M vs. $\mathrm{H}$ for $\left(\mathrm{Mo}_{2 / 3} \mathrm{~Tb}_{1 / 3}\right)_{2} \mathrm{AlC}$. Arrows indicate field-induced transitions. d. Magnetic scattering at $8 \mathrm{~K}$ obtained by subtracting the pattern at $100 \mathrm{~K}$ and its refinement. e. Magnetic scattering at $22 \mathrm{~K}$ obtained by subtracting the pattern at $100 \mathrm{~K}$ and its refinement. f. Schematic of magnetic structures at $8 \mathrm{~K}$ and $22 \mathrm{~K}$. Al atoms are not shown for clarity. 

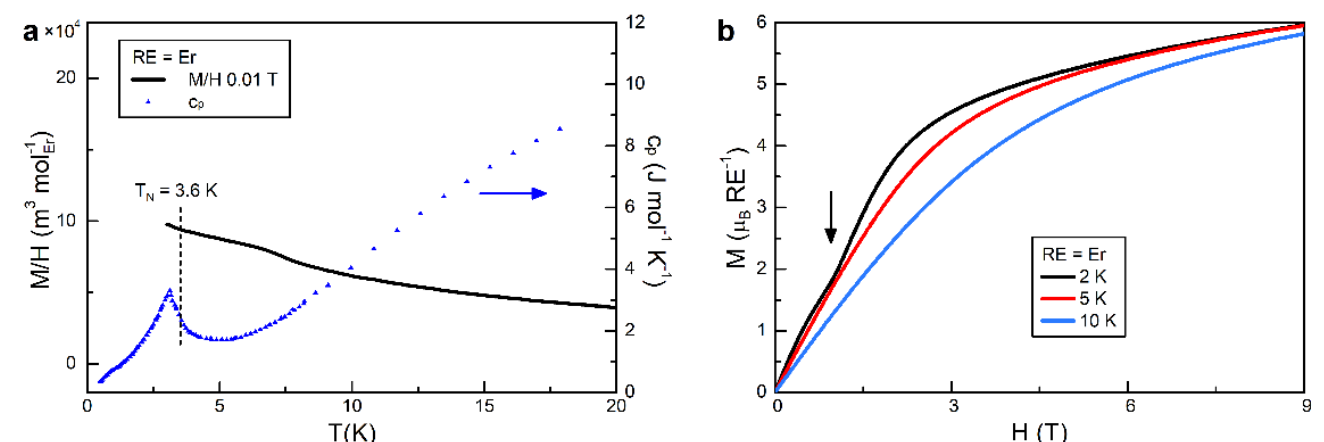

e
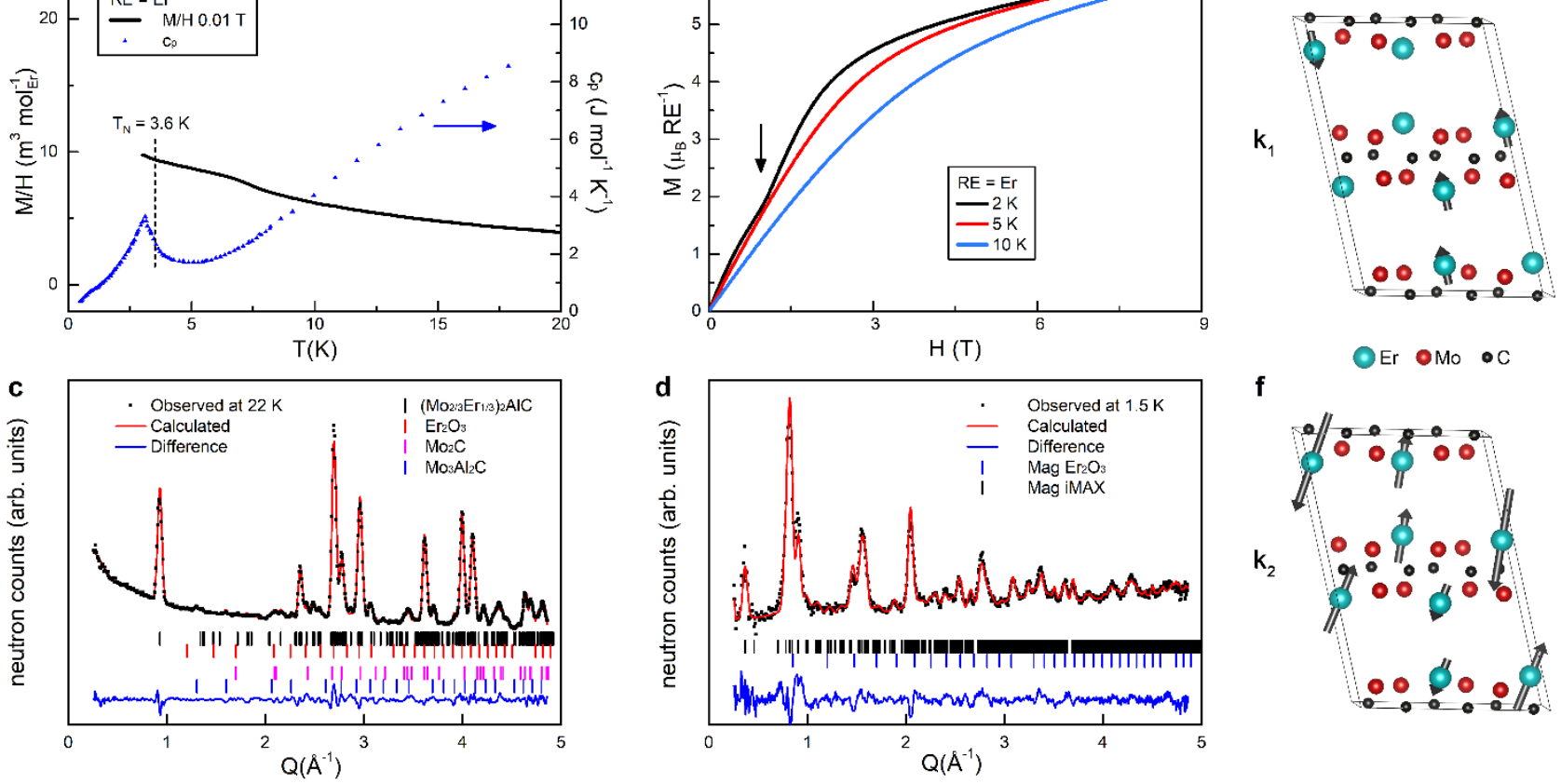

f

- Er OMo •C

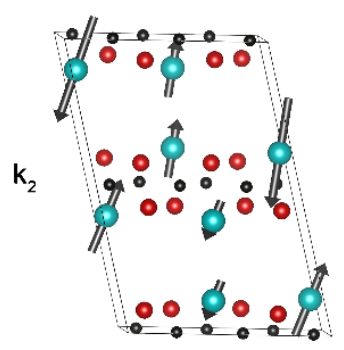

Fig. 7. Magnetic properties of $\left(\mathrm{Mo}_{2 / 3} \mathrm{Er}_{1 / 3}\right)_{2} \mathrm{AlC}$. a. M/H vs. T (left axis) and $\mathrm{c}_{\mathrm{p}} \mathrm{vs.} \mathrm{T}$ (right axis) for $\left(\mathrm{Mo}_{2 / 3} \mathrm{Er}_{1 / 3}\right)_{2} \mathrm{AlC}$. b. M vs. $\mathrm{H}$ at different temperatures. c. NPD above $\mathrm{T}_{\mathrm{N}}$ at $22 \mathrm{~K}$. d. Magnetic scattering at $1.5 \mathrm{~K}$. e-f. Schematic of the $\mathrm{k}_{1}$ and $\mathrm{k}_{2}$ magnetic structures are shown. 
Table 1. Parameters from Curie-Weiss fitting and magnetization measurements.

\begin{tabular}{|l|l|l|l|l|l|}
\hline $\mathrm{RE}$ & $\mathrm{T}_{\mathrm{N}}$ or $\mathrm{T}_{\mathrm{C}}(\mathrm{K})$ & $\Theta(\mathrm{K})$ & $\mu_{\text {eff }}\left(\mu_{\mathrm{B}}\right)$ & $\mathrm{M}_{9 \mathrm{~T}}\left(\mu_{\mathrm{B}}\right)$ & $p\left(\mu_{\mathrm{B}}\right)$ \\
\hline $\mathrm{Pr}$ & 17.0 & $4.3^{*}$ & $3.58^{*}$ & 1.50 & 3.58 \\
\hline $\mathrm{Nd}$ & 7.6 & -9.3 & 3.73 & 1.60 & 3.62 \\
\hline $\mathrm{Sm}$ & $\mathrm{N} / \mathrm{A}$ & $\mathrm{N} / \mathrm{A}$ & $\mathrm{N} / \mathrm{A}$ & 0.03 & 0.85 \\
\hline $\mathrm{Gd}$ & 26.0 & -20.3 & 8.34 & 2.14 & 7.94 \\
\hline $\mathrm{Tb}$ & $20.1 / 28.0$ & -12.1 & 9.70 & 3.40 & 9.72 \\
\hline $\mathrm{Dy}$ & $13.3 / 16.0$ & $-1.0^{*}$ & $10.51^{*}$ & 6.74 & 10.63 \\
\hline $\mathrm{Ho}$ & 7.8 & -4.5 & 10.28 & 5.60 & 10.60 \\
\hline $\mathrm{Er}$ & 3.6 & $7.5^{*}$ & $9.24^{*}$ & 6.06 & 9.59 \\
\hline $\mathrm{Tm}$ & N/A & $6.2^{*}$ & $7.32^{*}$ & 4.30 & 7.57 \\
\hline
\end{tabular}

${ }^{*}$ denotes that the values are obtained from $\mathrm{H} / \mathrm{M}$ vs. T. Otherwise they are obtained from $1 / \chi$ vs. T, in which $\chi$ is obtained from Arrott plot. $p(\mu \mathrm{B})$ was calculated according to Hund's rule. 
Insert Table of Contents artwork here

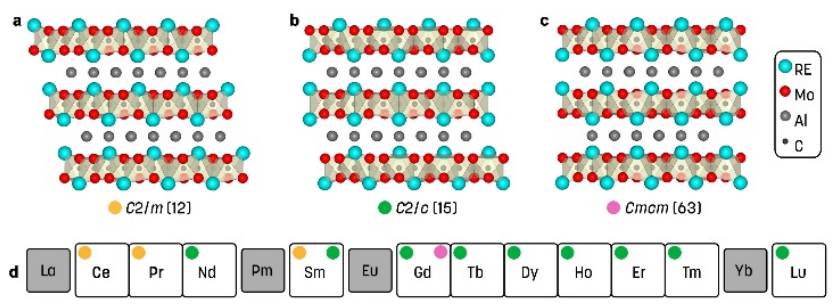

